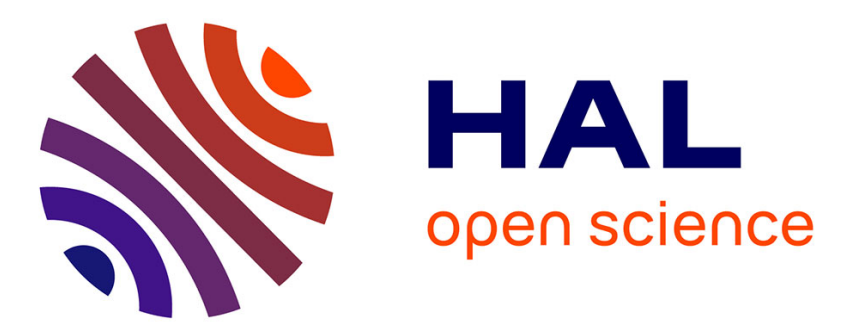

\title{
Magnetomechanical Damping and Mechanical Barkhausen Effect as Measured for Normal Load Mode
}

\author{
B. Augustyniak, M. Chmielewski, J. Chicois
}

\section{To cite this version:}

B. Augustyniak, M. Chmielewski, J. Chicois. Magnetomechanical Damping and Mechanical Barkhausen Effect as Measured for Normal Load Mode. Journal de Physique IV Proceedings, 1996, 06 (C8), pp.C8-541-C8-544. 10.1051/jp4:19968118 . jpa-00254548

\section{HAL Id: jpa-00254548 https://hal.science/jpa-00254548}

Submitted on 1 Jan 1996

HAL is a multi-disciplinary open access archive for the deposit and dissemination of scientific research documents, whether they are published or not. The documents may come from teaching and research institutions in France or abroad, or from public or private research centers.
L'archive ouverte pluridisciplinaire HAL, est destinée au dépôt et à la diffusion de documents scientifiques de niveau recherche, publiés ou non, émanant des établissements d'enseignement et de recherche français ou étrangers, des laboratoires publics ou privés. 


\title{
Magnetomechanical Damping and Mechanical Barkhausen Effect as Measured for Normal Load Mode
}

\author{
B. Augustyniak, M. Chmielewski and J. Chicois* \\ Department of Applied Physics, Technical University of Gdańsk, 80-952 Gdańsk, Poland \\ * GEMPPM, Bât. 502, INSA de Lyon, 69621 Villeurbanne, France
}

\begin{abstract}
Magnetomechanical hysteresis process was tested using normal mode of load. Tensile and compresion load with constant stress rate of $10 \mathrm{MPa} \mathrm{s}^{-1}$ was applied to the rod-like sample of the St 3 low carbon Polish steel (cross section $40 \mathrm{~mm}^{2}$ ). The intensiy of the mechanical Barkhausen noise (MBN) was measured during the first tensile load (up to $130 \mathrm{MPa}$ ) and during cyclic loading for various amplitudes of stress. The magnetomechanical energy losses (MEL) for one cycle of load was calculated from the magnetomechanical strain-stress hysteresis loops. There were established two features of MBN intensity: 1linear relation between the integral of the MBN intensity as calculated from the first load experiment and the integral of the MBN intensity hysteresis loop for a given stress amplitude and 2 - linear relation between MBN intensity hysterersis loop integral and the MEL integral for one cycle of load for the same stress amplitude. These results makes evident, that the MBN intensity is proportional to energy loss rate.
\end{abstract}

\section{INTRODUCTION}

Mechanical Barkhausen noise (MBN) effect is the mechanical analogue of classical Barkhausen effect and is produced when mechanical load is applied to the ferrous sample [1,2]. The change in applied stress produces reversible and irreversible abrupt motion of non- $180^{\circ}$ Bloch domain walls (DW) in the case of non-zero magnetostrictive materials [3]. Abrupt jump of such DW can be detected, as in the case of classical Barkhausen effect, by means of voltage signal induced in a search coil surrounding the loaded sample. The same process leads to the well known magnetomechanical internal friction (MIF). It seems to be evident, that MBN signal depicts magnetomechanical hysteresis process and should be closely related to the magneto-mechanical energy losses (MEL). This paper reports results of a new experiment that was performed to proof the linear dependence between MBN intensity and MEL rate. Such relation was deduced previously from the results of $\mathrm{MEL}$ and $\mathrm{MBN}$ analysis in the case of torsional vibration of wirelike samples [4]. It was found that the integral of the MBN intensity for one hysteresis loop was linearly dependent on the MEL integrated in one period of vibration. Such linear relation between MBN intensity and MEL rate was also confirmed indirectly by the result of the experiment with amorphous ribbons in which the internal stress $\sigma_{\mathrm{i}}$ level as evaluated from the stress dependence of the MBN intensity, was found to be well correlated with the $\sigma_{\mathrm{i}}$ parameter which was evaluated from the $\Delta E$ effect [5]. The torsion like load mode, which is mostly applied in pendulums, makes easy the evaluation of MEL level for one cycle of vibration (specially when MIF level is low: $Q^{-1} \approx 10^{-3}$ ) but such mode of load is not convenient for quantitative analysis of stress dependence of magnetomechanical damping because of the non-uniform stress distribution in the sample. It should be stressed, that MBN signal intensity is strongly attenuated and provides information only from near surface volume of stressed sample. That was the reason to perform the experiment in which the MBN intensity and the MEL level measurements have been done for a tensilecompression like mode of load with very low level of mechanical noise load machine. The MEL level for one cycle of load was evaluated directly from the magneto-mechanical strain-stress hysteresis loop. 


\section{EXPERIMENTAL RESULTS}

\subsection{Samples}

Polish low carbon St3 steel (concentration of C: $0.10 \mathrm{wt} \%$ ) samples of cross section $40 \mathrm{~mm}^{2}$ were used. The yield stress of that steel is $360 \mathrm{MPa}$, and the primary chemical impurity constituents are Mn (less then $1.65 \mathrm{wt} \%$ ), $\mathrm{Si}$ (less then $0.50 \mathrm{wt} \%$ ) and $\mathrm{P}$ and $\mathrm{S}$ (up to $0.045 \mathrm{wt} \%$ ). Before loading, the samples were annealed (argon atmosphere, $T \approx 800 \mathrm{~K}$ during $24 \mathrm{~h}$, slow cooling) in order to reduce residual stress level.

\subsection{Experimental conditions}

Loading machine, an oil-pressed push-pull like machine, was made by J. Chicois of INSA de Lyon [6,7]. The strain $\varepsilon$ was controlled with precision strain gages with accuracy better than $\Delta \varepsilon=0.1 \cdot 10^{-6}$. Load was applied using tension to stress converter with stress feedback and tension generator designed in Technical University of Gdansk. That generator provides triangular-like tension $U(t)$ that starts from $U(0)$ $=0$ level and varies with constant rate within the tuned range of maximal tension levels $\pm U_{\mathrm{o}}$. Sample was loaded within given stress amplitude $\sigma_{0}$ with a constant stress rate $\left(\mathrm{d} \sigma / \mathrm{d} t \approx 10 \mathrm{MPa} \mathrm{s}{ }^{-1}\right.$ ) and starting from $\sigma=0$ point. Before each loading the sample was demagnetised with a $\mathrm{C}$-shaped electromagnet [7]. The Barkhausen effect induced voltage measurements were made with a pickup coil of 200 turns wounded on the sample. The MBN voltage signal analyser was designed and made in Technical University of Gdańsk. The induced in pickup coil MBN voltage was amplified (total gain $K \approx 5 \cdot 10^{4}$ ), filtered with frequency band from $1 \mathrm{kHz}$ up to $200 \mathrm{kHz}$ and converted to output DC-like voltage signal with standard RMS analogue circuit. Data acquisition was via an analogue to digital (A/D) 12 bits PC card.

\subsection{MBN and MEL measurements}

The MBN intensity was measured 1) during "first load" monotonous increase of tensile stress up to the level $\sigma=130 \mathrm{MPa}$ and 2) - during tensile-compression cycling within given stress amplitude of stress $\sigma_{0}$. In the latter case the strain-stress hysteresis loops $a(\sigma)$ were also recorded. Fig. 1 shows the stress dependence of MBN intensity (in arbitrary units) as recorded during the "first load" of the sample. That plot reveals broad maximum of the MBN intensity. The highest level of MBN intensity appears at stress level $\sigma_{\mathrm{m}} \approx 40 \pm 5 \mathrm{MPa}$. It should be stressed, that the $\mathrm{MBN}$ intensity is also relatively high for the highest level of the applied stress. That means that effective internal stress barriers are higher then $130 \mathrm{MPa}$.

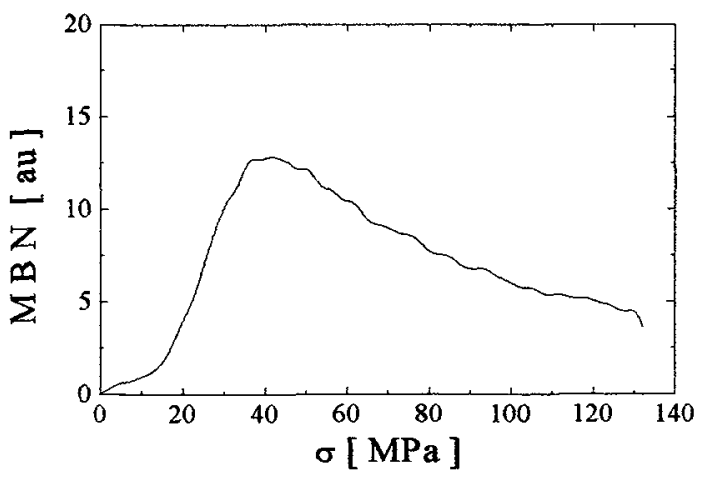

Fig. 1. Stress dependence of the MBN intensity recorded during the "first load".

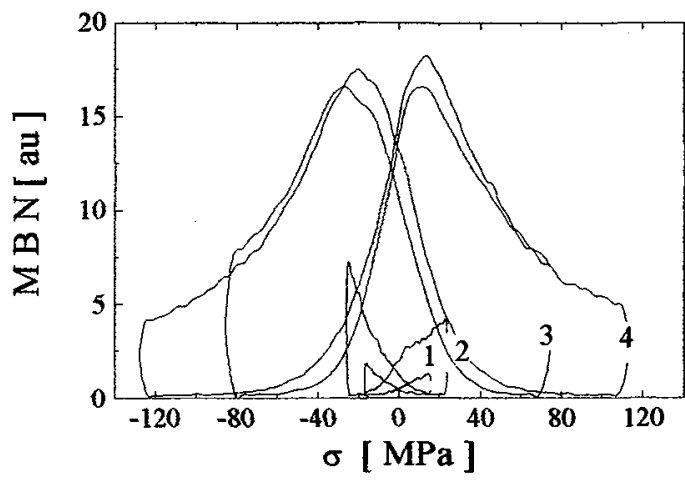

Fig.2. Hysteresis loops of MBN intensity recorded for various amplitudes of load. 
Fours examples of the MBN hysteresis loop as recorded during load cycling for various amplitudes of load are presented in Fig. 2. These plots reveal well defined butterfly-like hysteresis loop of MBN intensity. Its surface increases with $\sigma_{0}$ load amplitude and its shape depends on $\sigma_{0}$ level. The MBN intensity, as recorded during stress sweep between maximal stress values of strain $\sigma_{0}$, increases monotonously for low levels of $\sigma_{0}$. Maximum of MBN intensity appears at stress level of about $\mathrm{abs}(\sigma) \approx 20 \mathrm{MPa}$ for high levels of $\sigma_{0}$ amplitudes. Magneto-mechanical hysteresis process leads to stress-strain hysteresis loop phenomenon. That hysteresis loop is evaluated by means of subtraction of the $g(\sigma)$ hysteresis loop as measured without and with saturating magnetic field $H$, respectively. One example of the $\sigma(\sigma)$ hysteresis loop as recorded without magnetic field (for strain amplitude $\sigma_{0}=25 \mathrm{MPa}$ ) is shown in Fig. 3. One can find that strain hysteresis is equal $\Delta \varepsilon \approx 3.5 \cdot 10^{-6}$ (at $\sigma=0$ point of hysteresis loop).

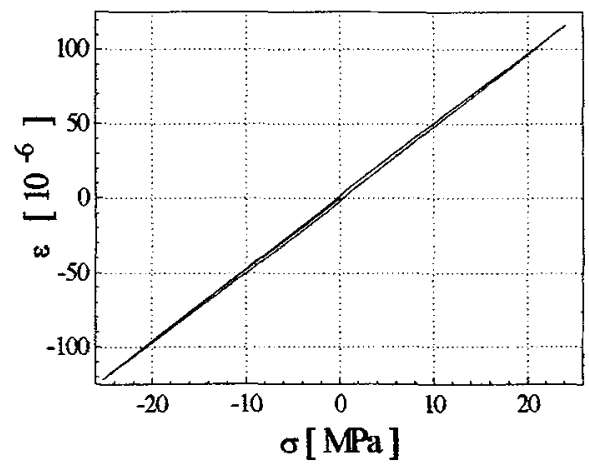

Fig. 3. Strain-stress hysteresis loop

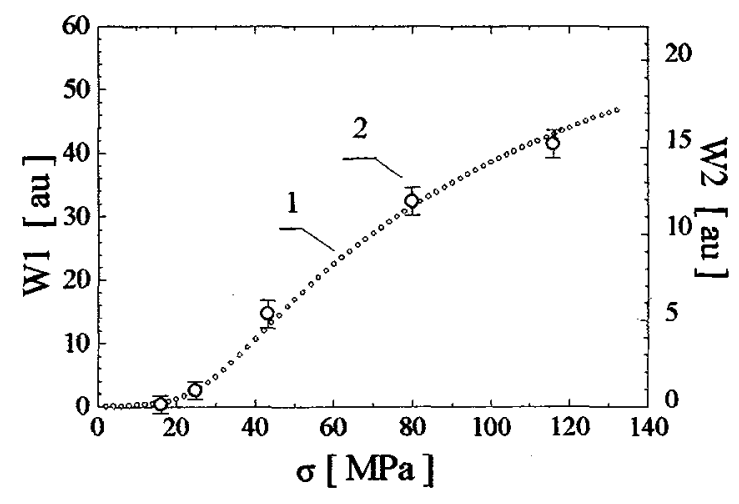

Fig. 4. Amplitude dependence of energy losses evaluated from :1 - "first load" - W1, 2 - hysteresis loop - W2

\section{DISCUSSION}

Some close relation between MBN intensity and MEL rate can be expected because the MBN effect reveals abrupt jumps of non- $180^{\circ} \mathrm{DW}$ and such Barkhausen jump provides local energy losses (mainly due to micro-eddy currents) and - leads to local supplementary and irreversible change of strain due to magnetostriction effect. For cycling load - magnetomechanical process leads thus to the strain-stress hysteresis loop as shown in Fig. 3. The area of that loop is equal to total mechanical energy losses $W$ in one cycle. The MBN intensity $U(\sigma)$ as measured for a given stress level depicts thus the energy dissipation process occurring at given stress level $\sigma$. That energy loss intensity can be described by $\mathrm{d} W(\sigma) / \mathrm{d} \sigma$ function, where $\mathrm{d} W(\sigma)$ is energy lose due to some $d \sigma$ increase of applied stress $\sigma$. The relation between MBN intensity and energy dissipation rate is assumed to be simply linear: $U(\sigma) \approx \mathrm{d} W(\sigma) / \mathrm{d} \sigma$. The MBN intensity depends obviously on stress rate $\mathrm{d} \sigma / \mathrm{d} t[8,9]$ and so in reported experiment load is applied with constant stress rate in order to exclude any corrections of the MBN intensity on stress rate. Verification of the assumed linear relation between MBN and MEL rate is based on analysis of the correlations between three parameters (called $W 1, W / 2$ and $W 3$ ) which are different evaluations of the magneto-mechanical energy losses in one cycle of load. These three parameters were evaluated from the "first load" MBN intensity $U(\sigma)$ function $(W 1)$, from $\mathrm{MBN}$ hysteresis loops $(W 2)$ and from magnetomechanical hysteresis loop (W3), respectively. The amplitude dependencies of these parameters should be linearly correlated each to other if the MBN intensity is proportional to energy losses rate. The first parameter $W 1$ it is the integral of the "first load" MBN $U(\sigma)$ stress dependence shown in Fig. 1 calculated within the stress range from 0 up to given $\sigma$ level. Such procedure is based on the experimentally confirmed relation between "first load" MBN $U(\sigma)$ function and internal stress distribution function $N(\sigma)$ [10]: $U(\sigma) \approx \sigma \cdot N(\sigma)[5,9]$. The internal stress distribution function $N(\sigma)$ is used for the magneto-mechanical hysteresis loop area evaluation by means of known expression: $W=\int \Delta W(\sigma)=\int W_{\mathrm{i}}, N(\sigma) \cdot \mathrm{d} \sigma$, where $W_{\mathrm{i}}$ is local magnetomechanical hysteresis loop area, which is assumed to be proportional to the stress amplitude $\sigma[10]$. 
This integral is calculated within amplitude range $0<\sigma<\sigma_{\mathrm{o}}$, where $\sigma_{\mathrm{o}}$ is given vibration amplitude. One can thus conclude that $\Delta W(\sigma) \approx U(\sigma) \cdot \mathrm{d} \sigma$ and evaluate the $W \cdot 1$ parameter as integral of $U(\sigma)$ function. The obtained $W 1(\sigma)$ dependence is shown in Fig. 4 by small points (plot 1). That plot reveals monotonous increase of $W 1$ parameter in function of stress $\sigma$. That dependence should be compared with amplitude dependence of total energy losses - parameter $W 2$, which is calculated as integral of $\mathrm{MBN}$ intensity hysteresis loops shown in Fig. 3. The resulting dependence $W 2(\sigma)$ is presented in Fig. 4 by open circles (plot 2). The expected close correlation between $W 1$ and $W 2$ parameters seems to be very well confirmed. The final test deals with correlation between $W 1$ and $W 2$ parameters and parameter $W 3$ which is the integral over the magneto-mechanical strain-stress hysteresis loop. Amplitude dependence of the calculated $W 3$ integrals is presented in Fig. 5 by solids circles (plot 2). There is also shown W1( $\sigma$ ) function (plot 1).

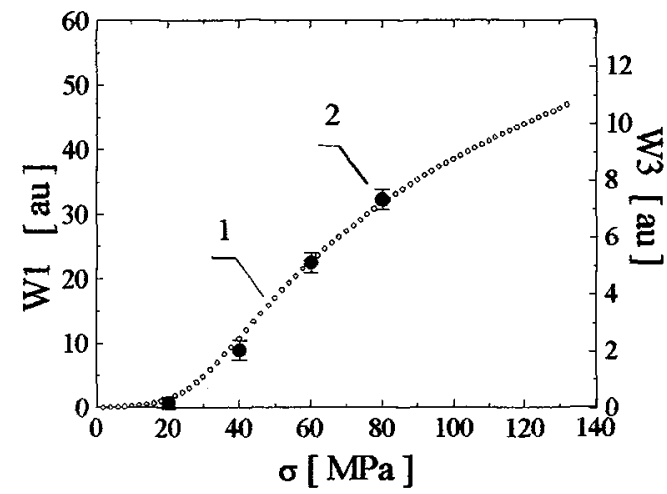

Fig. 5. Stress dependencies of $W 1$ (1) and $W 3$ (2) parameters

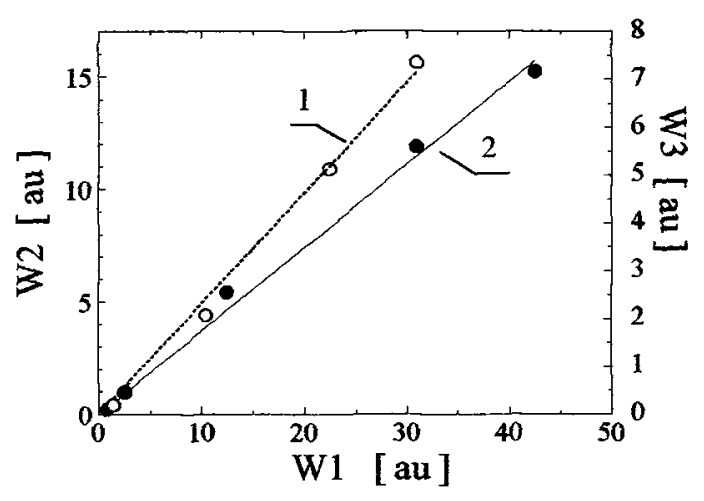

Fig. 6. Correlations between $W 2(1), W 3$ (2) parameters and $W 1$ parameter.

Fig. 5 depicts the same character of amplitude dependence of $W 1$ and $W 2$ parameters. Comparing Fig. 5 and Fig. 4 one can conclude that parameter $W 2$ and parameter $W 3$ are closely related. It is well confirmed by obvious linear plots as presented in Fig. 6 . These plots show intermediary relations between these two parameters and common $W 1$ parameter.

\section{CONCLUSIONS}

Two features of MBN intensity were found: 1- linear relation between the integral of the MBN intensity as calculated from the first load experiment and the integral of the MBN intensity hysteresis loop for a given stress amplitude and 2 - linear relation between MBN intensity hysteresis loop integral and the MEL integral for one cycle of load for the same stress amplitude. These results makes evident linear the relation between the MBN intensity and energy loss rate.

\section{References}

[1] Augustyniak B., Fantozzi G., Vannes A., J. de Phys. 46 (1985) C10-733.

[2] Augustyniak B., Vannes A., J. de Phys. 48 (1987) C8-407.

[3] Degauque J., Thése, Universite de Toulouse, France, 1977.

[4] Augustyniak B., Mater. Sc. Forum 119-121 (1993) 585-590.

[5] Małkiński L., Kaczkowski Z., Augustyniak B., J. of Magn. Magn. Mater., 112 (1992) 323-324.

[6] Chicois J., Thése, INSA de Lyon, France, 1987.

[7] Sablik M. J., Augustyniak B., J. Appl. Phys. 79 (1996) 963-972.

[8] Augustyniak B., Małkiński L., Molec. and Quantum Acoustic, 13 (1992) 189-196

[9] Augustyniak B, Chmielewski M., Proc. XVI Conf. Applied Crystallography, Cieszyn 1994, (World Scientific, Singapore 1995) p. 469-472.

[10] Birchak J.R, Smith G.W., J. Appl. Phys. 43 (1972) 1238-1245. 